\title{
T1 bladder carcinoma with variant histology: pathological features and clinical significance
}

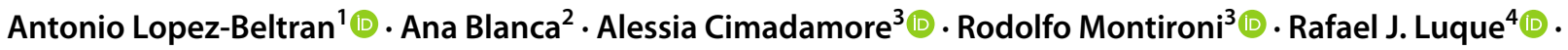 \\ Metka Volavšek ${ }^{5}$ (D) Liang Cheng ${ }^{6}$
}

Received: 29 October 2021 / Revised: 13 December 2021 / Accepted: 23 December 2021 / Published online: 4 February 2022

(c) The Author(s) 2022

\begin{abstract}
The aim of the study was to stratify high-grade T1 (HGT1) bladder urothelial carcinoma into risk categories based on the presence of variant histology when compared to conventional urothelial carcinoma. The clinicopathological features of 104 HGT1 cases of urothelial carcinoma of the bladder with variant histology present in 34 (37\%) were assessed. The endpoint of the study was disease-free survival and cancer-specific survival. Overall, variant histology was identified as a significant predictor of disease-free survival $(P=0.035)$. The presence of any specific variant histology (squamous, glandular, micropapillary, nested, microcystic, inverted growth, villous-like, basaloid, and lymphoepithelioma-like) was identified as a significant predictor of disease-free survival $(P=0.008)$ and cancer-specific survival $(P=0.0001)$ in HGT1 bladder cancer. Therefore, our results support including micropapillary HGT1 urothelial carcinoma within the aggressive high-risk category, as suggested by some recent clinical guidelines, but also favor nested, glandular, and basaloid to be placed in the high-risk category due to their potential of aggressive, life-threatening behavior and their limited response to bacillus Calmette-Guerin therapy. Conversely, the low-risk category would include urothelial carcinomas with squamous, inverted growth, or microcystic morphology, all with limited life-threatening potential and good response to current therapy. A very low-risk category would finally include patients whose tumors present villous-like or lymphoepithelioma-like morphology. In conclusion, our findings support the value of reporting the variant histology as a feature of variable aggressiveness in HGT1 urothelial carcinoma of the bladder.
\end{abstract}

Keywords Bladder $\cdot \mathrm{T} 1$ urothelial carcinoma $\cdot$ Variant histology $\cdot$ Biomarker $\cdot$ Staging

Antonio Lopez-Beltran

em1lobea@uco.es; em1lobea@gmail.com

1 Department of Morphological Sciences, University of Cordoba Medical School, Cordoba, Spain

2 Maimonides Biomedical Research Institute of Cordoba, E-14004 Cordoba, Spain

3 Institute of Pathological Anatomy and Histopathology, School of Medicine, Polytechnic University of the Marche Region, United Hospitals, Ancona, Italy

4 UGC Anatomía Patológica, Hospital Universitario de Jaén, Jaén, Spain

5 Institute of Pathology, Faculty of Medicine, University of Ljubljana, Ljubljana, Slovenia

6 Departments of Pathology and Laboratory Medicine and Urology, School of Medicine, Indiana University, Indianapolis, IN, USA

\section{Introduction}

Bladder carcinoma with variant histology represents about $20 \%$ of bladder urothelial carcinomas after TURBT (transurethral resection of bladder tumor) or cystectomy [1]. Most studies support variant histology as an aggressive feature with prognostic and therapeutic implications in advanced pT2-4 disease [2,3].

On the other hand, after standardized BCG (bacillus Calmette-Guerin) therapy, up to $75 \%$ of high-grade T1 (HGT1) disease patients will experience tumor recurrence within 2 years; up to $25 \%$ of them with a high risk of progression to muscle-invasive disease $[4,5]$. Hence, earlier identification of those tumors at risk of aggressive, life-threatening behavior would impact our practice. To accomplish this goal, it has been recommended that risk-associated features, such as tumor size, growth pattern and multifocality, extent/ depth of invasion, concomitant urothelial carcinoma in situ, 
lymphovascular invasion, and variant histology, be reported [6]. The role of variant histology to stratify patients at risk in HGT1 bladder carcinomas remains uncertain due to the limited number of related studies. Most reports on HGT1 with variant histology are reporting on micropapillary carcinoma or, rarely, nested carcinoma [4, 7-20]. The fact that some pathologists do not recognize or report about one-half of cases with variant histology in their practice is an additional limitation; therefore, the risk associated with variant histology in HGT1 carcinomas might indeed be underrecognized $[21,22]$.

To entangle the clinical situation, the AUA (American Urological Association) risk stratification for NMIBC (non-muscle-invasive bladder cancer) includes any variant histology as high risk; and the National Comprehensive Cancer Network (NCCN) guidelines support the inclusion of NMIBC with micropapillary, plasmacytoid, and sarcomatoid morphology as high risk of progression and death and recommends aggressive therapy. Some authors support early cystectomy in T1 micropapillary carcinoma to improve cancer-specific survival (CSS) [10, 23, 24]. Nonetheless, the appropriated management of HGT1 carcinomas with variant histology remains a matter of debate. Other relevant aspects to consider include the percentage of variant histology in cases of carcinoma with mixed histology, a parameter mainly investigated in micropapillary carcinoma, and the recent data in support of squamous and lymphoepitheliomalike morphology to predict the pathological response of antiPD-1 immunotherapy in a neoadjuvant setting relevant to the field of bladder preservation and to treat BCG-unresponsive HGT1 carcinomas [25-27]. Consequently, the ICCR dataset (International Collaboration on Cancer Reporting) recommends reporting and including an estimate of the variant histology present [6]. Thus, a risk classification associated with the different variant histology seen in HGT1 carcinoma would provide a rationale to stratify patients that can or cannot benefit from radical surgery (or other novel therapies), and therefore seems to be a necessary step of good practice in the management of these patients.

This paper aims to report the risk associated with variant histology in HGT1 bladder carcinoma compared with conventional urothelial carcinoma in a contemporary series.

\section{Materials and methods}

A total of 104 high-grade T1 bladder carcinoma samples were retrieved from the archives of Pathology Departments of participating institutions. Clinical information was obtained from medical records, and an average of three H\&E-stained slides from routine formalin-fixed and paraffin-embedded material (range, 1-6) from each case was systematically re-evaluated by three dedicated pathologists who confirmed the diagnosis and the presence of invasion to the level of subepithelial connective tissue. All cases were obtained by TURBT. Only primary cases with a negative second TURBT after the initial diagnosis were allowed. Cases with focally prominent discohesive features which occasionally imparted a plasmacytoid-like morphology were also excluded. Twelve cases were subsequently excluded upon review due to lack of relevant clinical information (seven cases lacked the applied BCG protocol or $<1$-year follow-up) or the presence of features supportive of muscularis propria invasion (two cases of micropapillary, one nested, and two plasmacytoid carcinomas). The remaining 92 cases were split into two groups: (i) cases with pure urothelial morphology classified as conventional urothelial carcinoma; (ii) cases with variant histology. In the latter category, except for urothelial carcinoma with squamous or glandular divergent differentiation, $\geq 50 \%$ of variant histology was required for inclusion. The presence of concomitant urothelial carcinoma in situ and focal tumor necrosis was also included in the study. All cases received BCG (bacillus Calmette-Guerin) treatment with maintenance, following the current clinical guidelines available at time of diagnosis, or mitomycin C (7.6\%). The cases spanned 14 years, with the earliest case diagnosed in 2002 and the latest in 2016, resulting in a follow-up of 13-170 months (mean $\pm \mathrm{SD}, 50.14 \pm 32.27$ ). Times to event for disease recurrence and cancer mortality were calculated from the date of TURBT to the date of last follow-up or death by cancer, and survival was measured from the date of the TURBT diagnosis to the date of last follow-up or death. Histological classification and depth of invasion of the tumors followed the 2016 revisions of WHO and AJCC, respectively [28, 29]. Recurrence was defined as finding new NMIBC after complete resection and induction course with $\mathrm{BCG}$, whereas progression was defined as the recurrence of a tumor with features of muscle-invasive bladder cancer after TURBT and BCG instillations.

Immunohistochemistry was performed on selected representative paraffin sections to resolve specific differential diagnostic issues required by the submitting pathologist. It included GATA3 (Cell Marque, clone L50-823, prediluted), CK20 (1:50, Dako, Glostrup, Denmark), MUC1 (Ventana, Clone H23, prediluted), p63 (1:100, Dako, Glostrup, Denmark), CK14 (Cell Marque, clone SP53, prediluted), CD3 (Cell Marque, rabbit polyclonal, prediluted), CK AE1AE3 (clone AE1/AE3, prediluted), CK-CAM 5.2 (1:2, Beckton, San Jose, CA), CK5/6 (clone 16B4; 1:50; Cell Marque, Rocklin, CA), or smoothelin (Cell Marque, clone R4A, prediluted). These were used as a single marker or as a combination thereof. Immunohistochemistry performance followed standard protocols for each antibody, using both Ventana-Benchmark or Leica Bond platforms and their 
respective reagents. Appropriate negative and positive controls were included in every run. Immunostaining was graded from 0 to $3+$, when appropriate.

Categorical variables were presented as frequencies and percentages and were compared using the $t$-test or chi-square test. The Kaplan-Meier method was used to estimate the distribution of survival separately for the categories with variant histology and conventional urothelial carcinoma. Differences among these two groups were tested for significance using the log-rank test (SPSS 15.0; SPSS, USA). A $P$-value $\leq 0.05$ was considered statistically significant.

\section{Results}

The main characteristics of the 92 cases of high-grade T1 bladder carcinoma with pure urothelial morphology or with variant histology (34 cases [37\%]) included nested or inverted growth carcinoma ( $8.7 \%$ each); micropapillary carcinoma (7.6\%); divergent differentiation (squamous [5.4\%] or glandular [2.2\%] carcinoma; and basaloid, microcystic, villous-like, or LELC (lymphoepithelioma-like) variants (1.1\% each) (Table 1; Figs. 1, 2, 3, 4). Eighty percent of patients were male with a mean age of 72.5 years (range, 38-92 years). Twenty-six (28.3\%) presented with concomitant urothelial carcinoma in situ or focal tumor necrosis (15 cases [16.3\%]), and $92.4 \%$ received BCG treatment. On a mean follow-up of 50 months (range, 13-170 months), $77.8 \%, 58.8 \%$, and $64.3 \%$ with conventional urothelial carcinoma recurred, progressed, or died of disease, respectively. Similarly, $22.2 \%, 41.2 \%$, and $35.7 \%$ with variant histology carcinoma recurred, progressed, or died of disease, respectively. Table 2 presents the univariate survival analysis in this series. There were differences between conventional urothelial carcinoma and urothelial carcinomas with variant histology $(P=0.035)$, and for specific variants $(P=0.008)$ regarding disease-free survival (DFS); and for CSS concerning variant subtype $(P=0.0001)$ (Fig. 5$)$. Gender status, concomitant urothelial carcinoma in situ, and the presence of tumor necrosis did not reach significance regarding DFS and CSS, with borderline significance regarding the response to BCG treatment $(P=0.069)$ (Table 2). Our results allowed a risk classification of HGT1 carcinoma based on the specific variant histology present (Fig. 6).

\section{Discussion}

Bladder carcinoma is morphologically heterogeneous, a process due to intratumoral heterogeneity and ultimately reflected by the presence of the variants of bladder cancer (variant histology) [30]. The current WHO revision includes urothelial carcinoma with divergent (squamous, glandular,
Table 1 Demography and clinicopathological characteristics of the 92 cases of T1 high-grade bladder carcinoma included in the study

\begin{tabular}{|c|c|}
\hline & $n(\%)$ \\
\hline Mean age (year) \pm SD (range) & $72.5 \pm 9.0(92-38)$ \\
\hline Mean follow-up (month) \pm SD (range) & $50.1 \pm 32.3(13-170)$ \\
\hline \multicolumn{2}{|l|}{ Gender } \\
\hline Female & $12(13.0)$ \\
\hline Male & $80(87.0)$ \\
\hline \multicolumn{2}{|l|}{ Variant histology } \\
\hline No & $58(63.0)$ \\
\hline Yes & $34(37.0)$ \\
\hline \multicolumn{2}{|l|}{ Variant subtype } \\
\hline Conventional urothelial carcinoma & $58(63.0)$ \\
\hline Nested & $8(8.7)$ \\
\hline Glandular & $2(2.2)$ \\
\hline Micropapillary & $7(7.6)$ \\
\hline Squamous & $5(5.4)$ \\
\hline Inverted & $8(8.7)$ \\
\hline Basaloid & $1(1.1)$ \\
\hline Microcystic & $1(1.1)$ \\
\hline Villous-like & $1(1.1)$ \\
\hline Lymphoepithelioma-like carcinoma & $1(1.1)$ \\
\hline \multicolumn{2}{|l|}{ Concomitant urothelial carcinoma in situ } \\
\hline No & $66(71.7)$ \\
\hline Yes & $26(28.3)$ \\
\hline \multicolumn{2}{|l|}{ Tumor necrosis } \\
\hline No & $77(83.7)$ \\
\hline Yes & $15(16.3)$ \\
\hline \multicolumn{2}{|l|}{ Bacillus Calmette-Guerin } \\
\hline No & $7(7.6)$ \\
\hline Yes & $85(92.4)$ \\
\hline \multicolumn{2}{|l|}{ Tumor recurrence } \\
\hline No & $65(70.7)$ \\
\hline Conventional urothelial carcinoma & $37(56.9)$ \\
\hline Variant histology & $28(43.1)$ \\
\hline Yes & $27(29.3)$ \\
\hline Conventional urothelial carcinoma & $21(77.8)$ \\
\hline Variant histology & $6(22.2)$ \\
\hline \multicolumn{2}{|l|}{ Tumor progression } \\
\hline No & $75(81.5)$ \\
\hline Conventional urothelial carcinoma & $48(64.0)$ \\
\hline Variant histology & $27(36.0)$ \\
\hline Yes & $17(18.5)$ \\
\hline Conventional urothelial carcinoma & $10(58.8)$ \\
\hline Variant histology & $7(41.18)$ \\
\hline \multicolumn{2}{|l|}{ Survival status } \\
\hline Alive & $65(70.7)$ \\
\hline Conventional urothelial carcinoma & $39(60.0)$ \\
\hline Variant histology & $26(40.0)$ \\
\hline Dead of bladder cancer & $14(15.2)$ \\
\hline Conventional urothelial carcinoma & $9(64.3)$ \\
\hline Variant histology & $5(35.7)$ \\
\hline
\end{tabular}


Table 1 (continued)

\begin{tabular}{ll}
\hline Dead of other cause & $\boldsymbol{n}(\%)$ \\
\hline
\end{tabular}

$S D$, standard deviation

and trophoblastic) differentiation, nested, microcystic, micropapillary, lymphoepithelioma-like (LELC), plasmacytoid/signet ring cell/diffuse, sarcomatoid, giant cell, poorly differentiated, lipid-rich, and clear cell (glycogen-rich) as histologic variants [28]. The presence of any variant or combinations thereof is considered high risk in NMIBC; [12] the NCCN clinical guidelines also recommend immediate radical cystectomy for HGT1 with micropapillary, plasmacytoid, or sarcomatoid variants, and the AUA guidelines of NMIBC include the presence of any histologic variant as a high-risk category $[31,32]$. The true incidence of variant histology in HGT1 urothelial carcinoma is uncertain, mainly based on limited reports of short case series, and ranges from 6.4 to $23 \%$ [33, 34]. Among HGT1 bladder cancer with variant histology, the micropapillary variant has received
Fig. 1 Conventional urothelial carcinoma seen at low (a) and high (b) power. Low- (c) and high-power (d) views of squamous differentiation. Glandular differentiation seen at low (e) and high power (f)
Fig. 2 Micropapillary carcinoma seen at low (a) and high (b) power. Low- (c) and high-power (d) views of nested carcinoma
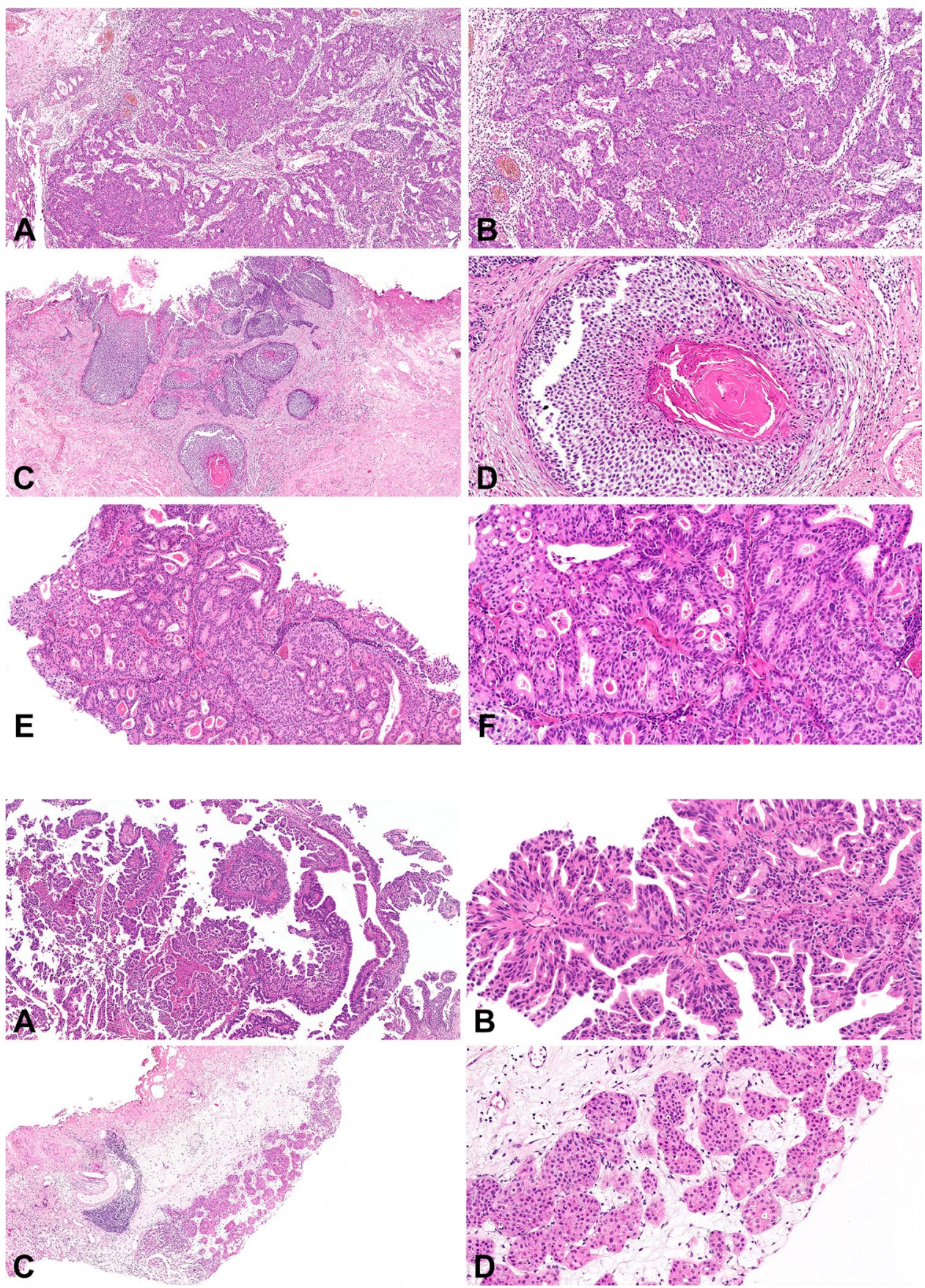
Fig. 3 Inverted urothelial carcinoma seen at low (a) and high (b) power. Low- (c) and highpower (d) views of basaloid carcinoma
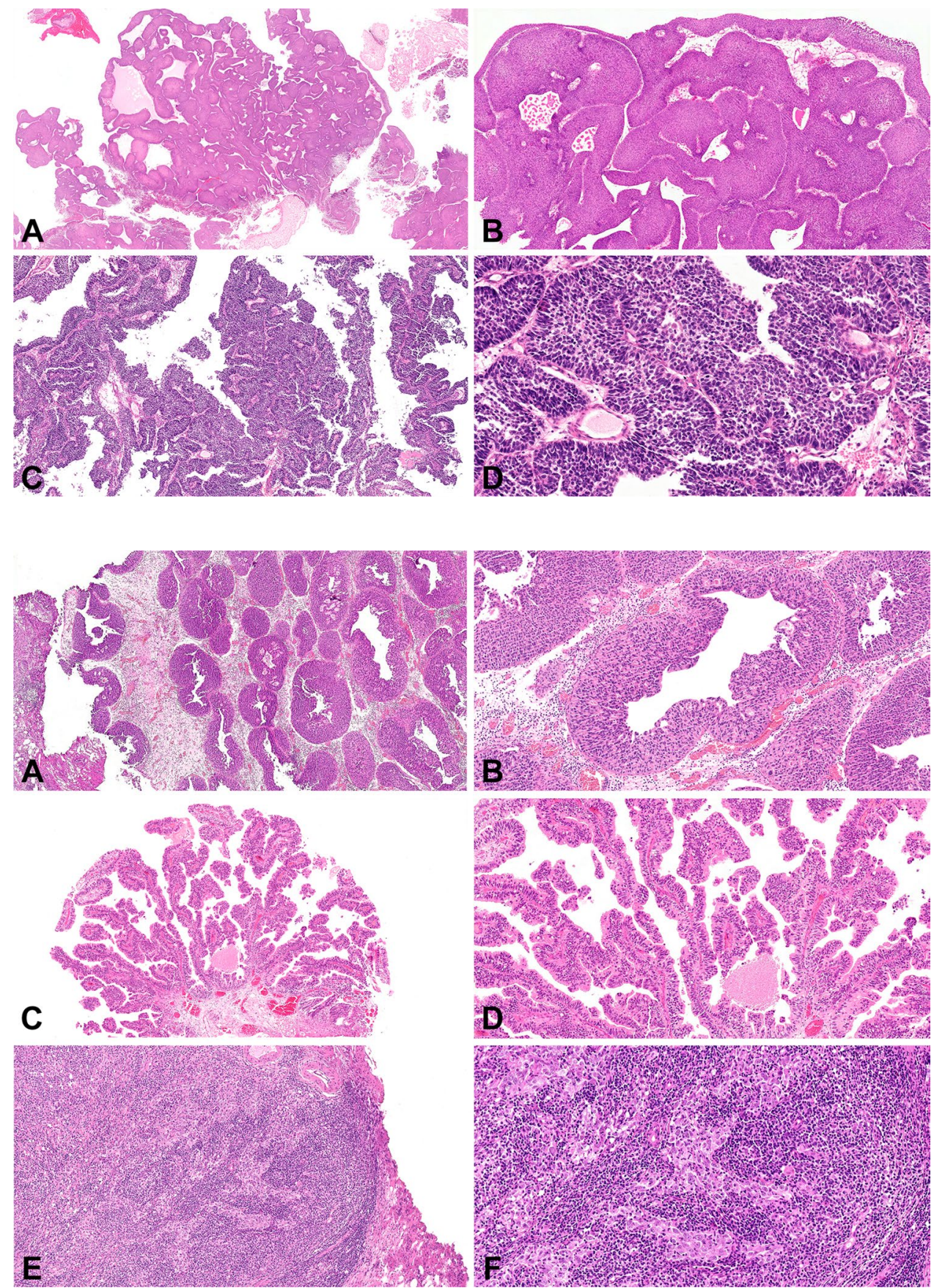

much attention, with $\mathrm{T} 1$ cases representing up to $40 \%$ of all-stage reported cases [9, 14, 22, 34-37]. T1 nested carcinoma represents $16 \%$ of all-stage reported cases $[9,20]$. Squamous divergent differentiation is also frequently seen in HGT1 carcinomas, with a reported rate of $14 \%$ [9, 22]. Nonetheless, data concerning other variants recognized by the WHO are limited mainly to small case series in HGT1, and their actual incidence remains largely unknown [22]. The substantial interobserver discordance when the central review of diagnostic cases is performed, and the fact that most variants are not recognized in daily practice by several pathologists, may explain the limited knowledge available on the risk associated with them in practice [21, 34].

Variant histology was associated with variable aggressive clinicopathological features in the current study, including survival and recurrence rate after complete TURBT and maintenance guided BCG instillations. Interestingly, the identified variants did not all show the same risk of aggressive disease. Squamous, glandular, micropapillary, nested, microcystic, and inverted growth variants showed lower DFS than villous-like, basaloid, and LELC, which showed no recurrences. On the other hand, micropapillary, 
Table 2 Univariate analysis for cancer-specific survival of parameters in the study using Kaplan-Meier plots and the Log-rank test

\begin{tabular}{|c|c|c|c|c|c|c|c|}
\hline & Overall $n=92$ & DFS & Log-rank & $P$-value & CSS & Log-rank & $P$-value \\
\hline Gender & & & 0.879 & 0.349 & & 0.531 & 0.466 \\
\hline Female & 12 & 4 & & & 2 & & \\
\hline Male & 80 & 23 & & & 12 & & \\
\hline Variant histology & & & 4.468 & 0.035 & & 0.156 & 0.693 \\
\hline No & 58 & 21 & & & 9 & & \\
\hline Yes & 34 & 6 & & & 5 & & \\
\hline Variant subtype & & & 15.126 & 0.008 & & 55.091 & 0.0001 \\
\hline Urothelial, not otherwise specified & 58 & 21 & & & 9 & & \\
\hline Nested & 8 & 1 & & & 2 & & \\
\hline Glandular & 2 & 1 & & & 1 & & \\
\hline Micropapillary & 7 & 0 & & & 1 & & \\
\hline Squamous & 5 & 0 & & & 0 & & \\
\hline Inverted & 8 & 3 & & & 0 & & \\
\hline Basaloid & 1 & 0 & & & 1 & & \\
\hline Microcystic & 1 & 1 & & & 0 & & \\
\hline Villous & 1 & 0 & & & 0 & & \\
\hline Lymphoepithelioma-like carcinoma & 1 & 0 & & & 0 & & \\
\hline Bacillus Calmette-Guerin & & & 0.239 & 0.625 & & 3.317 & 0.069 \\
\hline No & 7 & 1 & & & 2 & & \\
\hline Yes & 85 & 26 & & & 12 & & \\
\hline Concomitant urothelial carcinoma in situ & & & 0.01 & 0.920 & & & \\
\hline No & 66 & 20 & & & 10 & 0.008 & 0.931 \\
\hline Yes & 26 & 7 & & & & & \\
\hline Necrosis & & & 0.036 & 0.849 & & 0.605 & 0.437 \\
\hline No & 77 & 23 & & & 11 & & \\
\hline Yes & 15 & 4 & & & 3 & & \\
\hline
\end{tabular}

$D F S$, disease-free survival; CSS, cancer-specific survival

nested, glandular, and basaloid variants showed lower CSS rates than squamous, inverted, microcystic, villous-like, and LELC variants whose patients remained alive over 150 months of follow-up. Miyake et al., [12] in an extensive series of 1490 patients with NMIBC, recently reported an incidence of $6.4 \%$ with variant histology. These patients are more likely to result in cancer-related death than those with conventional urothelial carcinoma or with divergent differentiation, similar to our findings concerning risk stratification; in fact, our cases with divergent squamous differentiation were included as part of the low-risk category, and cases such as micropapillary and nested carcinomas remained as aggressive, life-threatening high-risk diseases. Likewise, Vourganti et al. [38], using data from the Surveillance, Epidemiology, and End Results (SEER) database, found no survival difference for micropapillary compared to conventional urothelial carcinoma after adjusting for stage and grade. Interestingly, these authors found similar behavior for cases reported as low-grade micropapillary NMIBC compared with high-grade micropapillary and high-grade urothelial carcinoma. This is an interesting point of practice, and a potential pitfall since rare cases of $\mathrm{T} 1$ micropapillary carcinomas may have bland cytology, and one may wonder if they should be categorized as low grade [38].

Currently, most publications on T1 urothelial carcinoma with variant histology have been dedicated to micropapillary or, rarely, to nested variants. However, our study additionally included a representation of other variants seen in HGT1 carcinomas with different sensitivities to recurrence and survival. This approach allowed us to identify a low- vs. high-risk classification associated with different variants. This includes micropapillary and nested, both reportedly associated with aggressive behavior [2, 35]. Consequently, our results support the inclusion of HGT1 carcinoma with micropapillary or nested features within the high-risk (aggressive) category. However, caution should be given to managing patients with micropapillary carcinomas since some studies suggest that these may be well controlled using standard TURBT and BCG therapy [14]. Therefore, it remains controversial if NMIBC with micropapillary features should undergo aggressive therapy ab initio due to some studies in which limited representation of superficial 

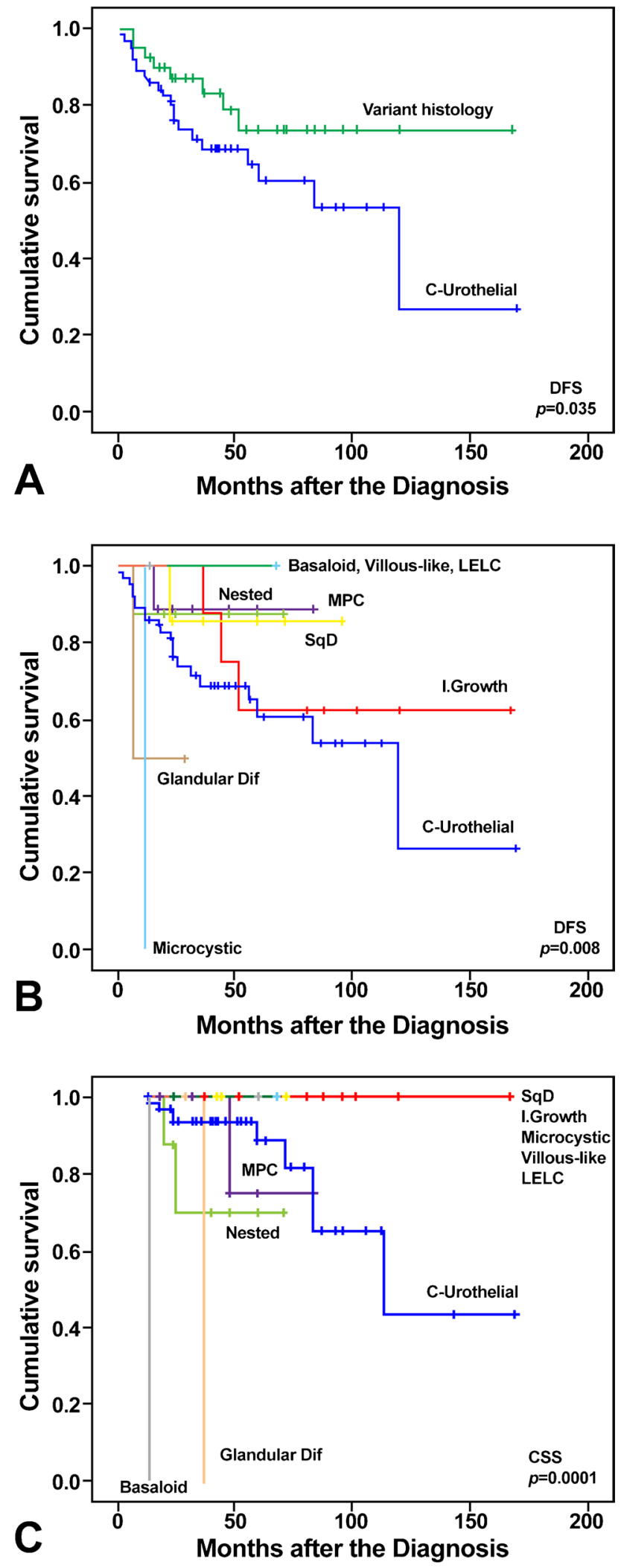

Fig. 5 Kaplan-Meier plots identify variant histology (a) and variant subtypes (b) as significant predictors of disease-free survival (DFS) in high-grade $\mathrm{T} 1$ carcinoma; variant subtypes were also significant predictors of cancer-specific survival (CSS) (c) micropapillary features (less than $10 \%$ micropapillary) was unrelated to aggressive behavior compared with conventional urothelial carcinoma; these cases should not be classified as micropapillary carcinoma [1, 15, 19, 25, 28, 37].

Our study also supports a subgroup of variants with low-risk features, which also agrees with the limited data reported in the current literature. The prognostic significance of variant histology in HGT1 tumors remains controversial; however, we hypothesized that our high-risk category follows similar significance as to the reported for variant histology in advanced bladder carcinomas in which variant histology seems to be related to poor behavior after current therapies. Our study, therefore, confirms the potential value of variant histology in risk assessment of HGT1 bladder cancer through different sensitivities regarding recurrence, progression, or survival associated with different histologic variants (see graphic representation, Fig. 6), although, our risk categorization is mostly based on cancer specific survival following guidelines supported therapy. A challenging point is that there were no cases of plasmacytoid carcinoma in our series, similar to Miyake's recent study of 1490 cases of HGT1 carcinoma; this is probably due to the rarity of the histologic variant in HGT1 carcinomas, with no reported series to date in the English literature [12, 39]. It seems reasonable to include plasmacytoid morphology as part of our high-risk category.

The retrospective nature of the current study and the fact that different pathologists selected the cases in different institutions with only one case each of the basaloid, microcystic, villous-like and lymphoepithelioma-like variants should be considered a limitation. However, our pathology-oriented series represents histologic variants other than those reported in clinically oriented series mainly limited to micropapillary and, to a lesser extent, to nested carcinomas [35, 40]. This is interesting since, clinical series exemplify the confusion associated with histologic variants by some clinical groups, but also exemplify the differences in reporting criteria from pathologists in different institutions; for instance, some of the reported series included only the presence of squamous or glandular divergent differentiation, while others included only micropapillary or nested variants with the concept of variants $[15,35,41]$. To avoid entity-derived limitations, we have conducted an accurate review of all pathologic materials, following classic diagnostic criteria for urothelial carcinomas updated to include the terminology and subtypes recognized by the current WHO classification of urogenital tumors, as well as recently published data [1, $3,28]$. Additionally, our cases were diagnosed based on specialized genitourinary pathological assessment using contemporary criteria, which can also explain the inclusion of some rarer examples of variant histology not recognized by the current WHO classification (inverted growth, 
Fig. 6 The observed risk of aggressive disease, high-grade $\mathrm{T} 1$ urothelial carcinoma, allows to separate variant subtypes into low- (left) and high-risk (right) categories. A very low-risk category may be considered for villous and lymphoepitheliomalike (bottom left)

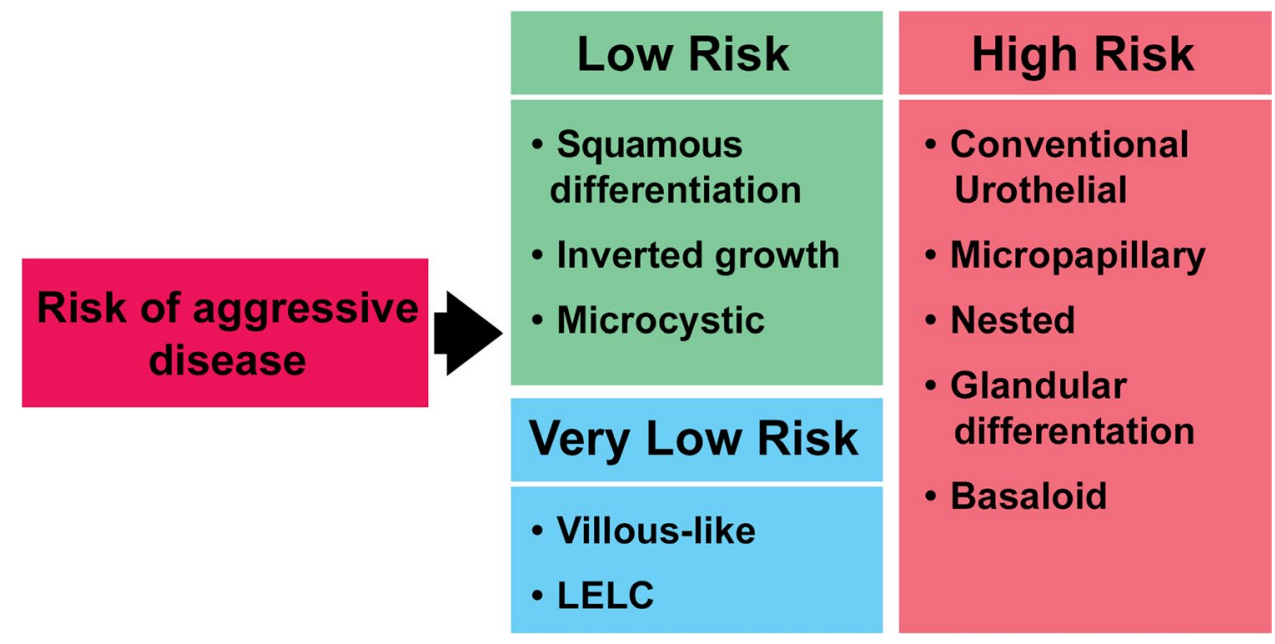

villous-like and basaloid carcinomas) [42]. In a context in which pathologic prognostic assessment is essential in HGT1 carcinoma management, reporting the presence and extension (i.e., percentage) of histologic variants seems to be essential as an element of good clinical practice.

In conclusion, we were able to identify a series of highgrade $\mathrm{T} 1$ bladder carcinomas in which the presence of specific variants offered further predictive information in this highly heterogeneous tumor, and that the disease control is likely achieved with a combination of complete TURBT and BCG therapy applied following the current guidelines. Consequently, patients with micropapillary, nested, or basaloid morphology or glandular divergent differentiation carcinoma should be considered in the context of the high-risk disease since they are potentially lifethreatening progressive tumors with variable-to-limited responses to therapy. The presence of divergent squamous differentiation, inverted growth, microcystic, and villouslike or LELC morphology should be considered within the low-risk disease with variable tumor recurrence and very low progression to life-threatening disease. If confirmed in a more extensive series or well-controlled clinical trials, our variant histology proposed risk stratification could be practice-changing in managing HGT1 urothelial carcinomas.

Author contribution ALB drafted the article. ALB, AC, RM, MV, and $\mathrm{LC}$ formulated and designed the study. $\mathrm{AC}, \mathrm{MV}$, and $\mathrm{AB}$, made the literature search. AB and RJL completed the statistical analysis. All the authors critically read, edited, and approved the final manuscript.

Funding Open Access funding provided thanks to the CRUE-CSIC agreement with Springer Nature. This study was supported in part by the Grant PI17/01981 [FIS (Ministry of Health), Madrid, Spain] (ALB and $\mathrm{AB}$ ).

\section{Declarations}

Competing interests The authors declare no competing interests.

Open Access This article is licensed under a Creative Commons Attribution 4.0 International License, which permits use, sharing, adaptation, distribution and reproduction in any medium or format, as long as you give appropriate credit to the original author(s) and the source, provide a link to the Creative Commons licence, and indicate if changes were made. The images or other third party material in this article are included in the article's Creative Commons licence, unless indicated otherwise in a credit line to the material. If material is not included in the article's Creative Commons licence and your intended use is not permitted by statutory regulation or exceeds the permitted use, you will need to obtain permission directly from the copyright holder. To view a copy of this licence, visit http://creativecommons.org/licenses/by/4.0/.

\section{References}

1. Lopez-Beltran A, Henriques V, Montironi R, Cimadamore A, Raspollini MR, Cheng L (2019) Variants and new entities of bladder cancer. Histopathology 74:77-96. https://doi.org/10.1111/his. 13752

2. Porten SP, Willis D, Kamat AM (2014) Variant histology: role in management and prognosis of nonmuscle invasive bladder cancer. Curr Opin Urol 24:517-523. https://doi.org/10.1097/MOU.00000 00000000089

3. Lopez-Beltran A, Cheng L (2006) Histologic variants of urothelial carcinoma: differential diagnosis and clinical implications. Hum Pathol 37:1371-1388. https://doi.org/10.1016/j.humpath.2006.05. 009

4. Jordan B, Meeks JJ (2019) T1 bladder cancer: current considerations for diagnosis and management. Nat Rev Urol 16:23-34. https://doi.org/10.1038/s41585-018-0105-y

5. Magers MJ, Lopez-Beltran A, Montironi R, Williamson SR, Kaimakliotis HZ, Cheng L (2019) Staging of bladder cancer. Histopathology 74:112-134. https://doi.org/10.1111/his.13734

6. Varma M, Srigley JR, Brimo F, Comperat E, Delahunt B, Koch $M$ et al (2020) Dataset for the reporting of urinary tract carcinoma-biopsy and transurethral resection specimen: recommendations from the International Collaboration on Cancer Reporting (ICCR). Mod Pathol 33:700-712. https://doi.org/10.1038/ s41379-020-0514-3 
7. Deuker M, Franziska Stolzenbach L, Rosiello G, Luzzago S, Martin T, Tian Z et al (2021) Radical cystectomy improves survival in patients with stage $\mathrm{T} 1$ squamous cell carcinoma and neuroendocrine carcinoma of the urinary bladder. Eur J Surg Oncol 47:463-469. https://doi.org/10.1016/j.ejso.2020.05.006

8. Esrig D, Freeman JA, Stein JP, Skinner DG (1997) Early cystectomy for clinical stage T1 transitional cell carcinoma of the bladder. Semin Urol Oncol 15:154-160

9. Gofrit ON, Yutkin V, Shapiro A, Pizov G, Zorn KC, Hidas G et al (2016) The response of variant histology bladder cancer to intravesical immunotherapy compared to conventional cancer. Front Oncol 6:43. https://doi.org/10.3389/fonc.2016.00043

10. Kamat AM, Gee JR, Dinney CP, Grossman HB, Swanson DA, Millikan RE et al (2006) The case for early cystectomy in the treatment of nonmuscle invasive micropapillary bladder carcinoma. J Urol 175:881-885. https://doi.org/10.1016/S00225347(05)00423-4

11. Mannas MP, Lee T, Nykopp TK, Batista da Costa J, Black PC (2018) A risk-stratified approach to the management of high-grade T1 bladder cancer. Curr Opin Urol 28:563-569. https://doi.org/10. 1097/MOU.0000000000000548

12. Miyake M, Nishimura N, Iida K, Fujii T, Nishikawa R, Teraoka S et al (2021) Intravesical Bacillus Calmette-Guerin treatment for T1 high-grade non-muscle invasive bladder cancer with divergent differentiation or variant morphologies. Cancers (Basel) 26:13. https:// doi.org/10.3390/cancers13112615

13. Nassiri N, Ghodoussipour S, Maas M, Nazemi A, Asanad K, Pearce $S$ et al (2020) Occult nodal metastases in patients down-staged to nonmuscle invasive disease following neoadjuvant chemotherapy. Urology 142:155-160. https://doi.org/10.1016/j.urology.2020.03. 021

14. Spaliviero M, Dalbagni G, Bochner BH, Poon BY, Huang H, AlAhmadie HA et al (2014) Clinical outcome of patients with T1 micropapillary urothelial carcinoma of the bladder. J Urol 192:702_ 707. https://doi.org/10.1016/j.juro.2014.02.2565

15. Willis DL, Fernandez MI, Dickstein RJ, Parikh S, Shah JB, Pisters LL et al (2015) Clinical outcomes of cT1 micropapillary bladder cancer. J Urol 193:1129-1134. https://doi.org/10.1016/j.juro.2014. 09.092

16. Zhao G, Wang C, Tang Y, Liu X, Liu Z, Li G et al (2019) Glandular differentiation in pT1 urothelial carcinoma of bladder predicts poor prognosis. Sci Rep 9:5323. https://doi.org/10.1038/ s41598-019-41844-4

17. Li Q, Assel M, Benfante N, Pietzak E, Bagrodia A, Cha E et al (2017) Clinical outcomes in patients with panurothelial carcinoma treated with radical nephroureterectomy following cystectomy for metachronous recurrence. J Urol 198:546-551

18. Li Q, Assel M, Benfante NE, Pietzak EJ, Herr HW, Donat M et al (2019) The impact of plasmacytoid variant histology on the survival of patients with urothelial carcinoma of bladder after radical cystectomy. Eur Urol Focus 5:104-108. https://doi.org/10.1016/j.euf.2017. 06.013

19. Lobo N, Shariat SF, Guo CC, Fernandez MI, Kassouf W, Choudhury A et al (2020) What is the significance of variant histology in urothelial carcinoma? Eur Urol Focus 6:653-663. https://doi.org/10. 1016/j.euf.2019.09.003

20. Lopez-Beltran A, Cheng L, Montironi R, Blanca A, Leva M, Roupret $M$ et al (2014) Clinicopathological characteristics and outcome of nested carcinoma of the urinary bladder. Virchows Archiv 465:199-205. https://doi.org/10.1007/s00428-014-1601-y

21. Shah RB, Montgomery JS, Montie JE, Kunju LP (2013) Variant (divergent) histologic differentiation in urothelial carcinoma is under-recognized in community practice: impact of mandatory central pathology review at a large referral hospital. Urol Oncol 31:1650-1655. https://doi.org/10.1016/j.urolonc.2012.04.009
22. Baumeister P, Zamboni S, Mattei A, Antonelli A, Simeone C, Mordasini L et al (2019) Histological variants in non-muscle invasive bladder cancer. Transl Androl Urol 8:34-8. https://doi.org/10.21037/ tau.2019.01.09

23. Monn MF, Kaimakliotis HZ, Pedrosa JA, Cary KC, Bihrle R, Cheng L et al (2015) Contemporary bladder cancer: variant histology may be a significant driver of disease. Urol Oncol 33(18):e5-e20. https:// doi.org/10.1016/j.urolonc.2014.10.001

24. Kamat AM, Dinney CP, Gee JR, Grossman HB, Siefker-Radtke AO, Tamboli P et al (2007) Micropapillary bladder cancer: a review of the University of Texas M. D. Anderson Cancer Center experience with 100 consecutive patients. Cancer 110:62-67. https://doi.org/ 10.1002/cncr.22756

25. Samaratunga H, Khoo K (2004) Micropapillary variant of urothelial carcinoma of the urinary bladder; a clinicopathological and immunohistochemical study. Histopathology 45:55-64. https://doi.org/10. 1111/j.1365-2559.2004.01895.x

26. Necchi A, Raggi D, Gallina A, Madison R, Colecchia M, Luciano $R$ et al (2020) Updated results of PURE-01 with preliminary activity of neoadjuvant pembrolizumab in patients with muscle-invasive bladder carcinoma with variant histologies. Eur Urol 77:439-446. https://doi.org/10.1016/j.eururo.2019.10.026

27. Necchi A, Raggi D, Gallina A, Ross JS, Fare E, Giannatempo P et al (2020) Impact of molecular subtyping and immune infiltration on pathological response and outcome following neoadjuvant pembrolizumab in muscle-invasive bladder cancer. Eur Urol 77:701-710. https://doi.org/10.1016/j.eururo.2020.02.028

28. Moch H, Humphrey PA, Ulbright TM, Reuter V (2016) WHO classification of tumours of the urinary system and male genital organs. 4th ed. Lyon, France: International Agency for Research on Cancer (IARC).

29. Amin M, Edge SB, Greene FL, Byrd DR, Brookland R, Washington $\mathrm{M}$ et al (2017) AJCC cancer staging manual, 8th edn. Springer, New York, NY

30. Meeks JJ, Al-Ahmadie H, Faltas BM, Taylor JA 3rd, Flaig TW, DeGraff DJ et al (2020) Genomic heterogeneity in bladder cancer: challenges and possible solutions to improve outcomes. Nat Rev Urol 17:259-270. https://doi.org/10.1038/s41585-020-0304-1

31. Chang SS, Boorjian SA, Chou R, Clark PE, Daneshmand S, Konety $\mathrm{BR}$ et al (2016) Diagnosis and treatment of non-muscle invasive bladder cancer: AUA/SUO guideline. J Urol 196:1021-1029. https:// doi.org/10.1016/j.juro.2016.06.049

32. Flaig TWe (2020). Bladder cancer, version 3.2020, NCCN Clinical Practice Guidelines in Oncologyhttps://doi.org/10.6004/jnccn.2020. 0011

33. Ploeg M, Aben KK, Hulsbergen-van de Kaa CA, Schoenberg MP, Witjes JA, Kiemeney LA (2010) Clinical epidemiology of nonurothelial bladder cancer: analysis of the Netherlands Cancer Registry. J Urol 183:915-920. https://doi.org/10.1016/j.juro.2009.11.018

34. Seisen T, Comperat E, Leon P, Roupret M (2014) Impact of histological variants on the outcomes of nonmuscle invasive bladder cancer after transurethral resection. Curr Opin Urol 24:524-531

35. Mally AD, Tin AL, Lee JK, Satasivam P, Cha EK, Donat SM et al (2017) Clinical outcomes of patients With T1 nested variant of urothelial carcinoma compared to pure urothelial carcinoma of the bladder. Clin Genitourin Cancer 14:30199-30204

36. Lopez-Beltran A, Montironi R, Blanca A, Cheng L (2010) Invasive micropapillary urothelial carcinoma of the bladder. Hum Pathol 41:1159-1164

37. Amin A, Epstein JI (2012) Noninvasive micropapillary urothelial carcinoma: a clinicopathologic study of 18 cases. Hum Pathol 43:2124-2128. https://doi.org/10.1016/j.humpath.2012.04.013

38. Vourganti S, Harbin A, Singer EA, Shuch B, Metwalli AR, Agarwal PK (2013) Low grade micropapillary urothelial carcinoma, does it exist?-Analysis of Management and Outcomes from the Surveillance, Epidemiology and End Results (SEER) database. J Cancer 4:336-342. https://doi.org/10.7150/jca.6215 
39. Sood S, Paner GP (2019) Plasmacytoid urothelial carcinoma: an unusual variant that warrants aggressive management and critical distinction on transurethral resections. Arch Pathol Lab Med 143:1562-1567. https://doi.org/10.5858/arpa.2018-0139-RS

40. Mitra AP, Fairey AS, Skinner EC, Boorjian SA, Frank I, Schoenberg MP et al (2019) Implications of micropapillary urothelial carcinoma variant on prognosis following radical cystectomy: a multi-institutional investigation. Urol Oncol 37:48-56. https://doi.org/10.1016/j. urolonc.2018.10.013

41. Kim SP, Frank I, Cheville JC, Thompson RH, Weight CJ, Thapa P et al (2012) The impact of squamous and glandular differentiation on survival after radical cystectomy for urothelial carcinoma. J Urol 188:405-409. https://doi.org/10.1016/j.juro.2012.04.020

42. Lim M, Adsay NV, Grignon D, Osunkoya AO (2009) Urothelial carcinoma with villoglandular differentiation: a study of 14 cases. Mod Pathol 22:1280-1286. https://doi.org/10.1038/modpathol.2009.97

Publisher's Note Springer Nature remains neutral with regard to jurisdictional claims in published maps and institutional affiliations. 